\title{
EDITORIAL
}

\section{Data Availability Principles and Practice}

\section{KEYWORD: Editorial}

Science requires evidence. Making data available lets other scientists confirm results, uncover errors, or find new insights. Moreover, gathering data can be expensive and time consuming. Since the same data can be used for a range of purposes, making data available can be an efficient use of limited research resources. Doing so can also improve traceability and, thus, accountability, when it comes to research findings.

These reasons and more lie behind recent efforts to promote data availability. The American Meteorological Society (AMS) recently updated its data policy guidelines (https://www.ametsoc.org/ index.cfm/ams/publications/ethical-guidelines-and-ams-policies/data-policy-and-guidelines/) to require, among other things, that papers in its journals include a Data Availability Statement. Data do not need to be "open." Authors simply need to explain how to find and use the data or why, in some limited circumstances, the data are not available.

Papers that appear in Journal of Atmospheric and Oceanic Technology (JTECH) involve data of different natures, ranging from large observational datasets to small datasets generated for instrument testing that could be of limited interest to other scientists. The Data Availability Statement should make clear what has been archived and what steps have been taken to provide information about the data that could not be kept. We do not wish to impose a "one size fits all" requirement that may serve as a barrier to authors. In fact, one of our goals in this endeavor is to ensure that no authors are excluded from publishing in our journals because of resource limitations, institutional restrictions on sharing data, or legal constraints.

The Data Availability Statement need not be long, and the statement does not count toward the word count limit. If data are for some reason unavailable, authors must make a good-faith effort to explain the circumstances. See the AMS example statements to gain a sense of expectations (https:// www.ametsoc.org/index.cfm/ams/publications/authors/journal-and-bams-authors/formatting-and-manuscriptcomponents/data-availability-statement-examples/). Our editors are willing to work with authors to accommodate any difficulties or unique situations.

Thoughtful data availability requirements such as AMS's benefit both the scientific community and society. Consistent policies and practices can help to reduce misunderstanding and divergent interpretations. As editors, we do not wish or intend that the data availability requirement become a barrier to publication, whether because of the sensitivity of the data or because of limited institutional resources. At the same time, the exceptions to making data available should not be used by researchers as a way to evade their responsibilities. We welcome contributors to and readers of JTECH to read the AMS data policy and to contact us with any questions or concerns.

Luca Baldini William Emery Co-Chief Editors

D Denotes content that is immediately available upon publication as open access. 